SUPPORTING INFORMATION

\title{
Detection of Opioids on Mail/Packages using Open Port Interface Mass Spectrometry (OPI-MS)
}

\author{
Haidy Metwally ${ }^{1}$, Prashant Agrawal ${ }^{1}$, Rachael Smith ${ }^{1}$, Chang Liu ${ }^{2}$, Yves LeBlanc ${ }^{2}$ \\ Thomas R. Covey ${ }^{2}$, and Richard Oleschuk ${ }^{1 *}$ \\ ${ }^{1}$ Department of Chemistry, Queen's University, Kingston, Ontario, \\ K7L 3N6, Canada. \\ ${ }^{2}$ SCIEX, 71 Four Valley Drive, Concord, Ontario L4K 4 V8, Canada \\ E-mail address of the corresponding author: richard.oleschuk@chem.queensu.ca
}

* Corresponding author: Richard Oleschuk

This file contains:

Materials and Methods

Supporting Figures 


\section{Materials and Methods}

Materials. All solvents used (methanol, acetonitrile, and formic acid) were purchased from Fischer Scientific (Ottawa, ON). Ziploc bags ${ }^{\mathrm{TM}}$ were purchased locally and manufactured by S.C. Johnson and son (Brantford, ON). Coffee used in the surrogate experiment was obtained from a local coffee shop at Queen's University, house blend.

\section{Mass Spectrometry.}

An OPI was externally connected via PEEK tubing (1/16" OD*0.01" ID, IDEX, NY, USA) to an API 3200 triple quadrupole mass spectrometer (Sciex, Concord, ON) and its electrospray ionization source (ESI). The ESI probe was maintained at a voltage of $+5.5 \mathrm{kV}$, with ion source nitrogen gas settings $\mathrm{GS} 1=45 \mathrm{psi}, \mathrm{GS} 2=20 \mathrm{psi}$, curtain gas $=50 \mathrm{psi}$ and heated nebulizer temperature $=300{ }^{\circ} \mathrm{C}$. The OPI is connected to an external syringe pump for solvent delivery. The flow rate of solvent ( $50 \%$ methanol or acetonitrile: $49.9 \%$ water: $0.1 \%$ formic acid) was controlled by the aspiration force induced by GS1 (nebulizing gas) to achieve a constant dome-shaped sampling surface without over-spilling. Tandem mass spectrometry (MS/MS) conditions were declustering potential $(\mathrm{DP})=45 \mathrm{~V}$, entrance potential $(\mathrm{EP})=10 \mathrm{~V}$, collision energy $(\mathrm{CE})=32 \mathrm{~V}$, collision cell exit potential $(\mathrm{CXP})=10$.

Data Analysis. All of the curve fitting calculations were done using Sigmaplot 11.0 while the limits of mass detection ( $\mathrm{LOD}_{\text {mass }}$ ) were computed in excel by employing linear regression method:

$$
L O D_{\text {mass }}=\frac{3 S}{b}
$$

$S$ : is the standard deviation of the response, while $b$ is the slope of the calibration curve. ${ }^{1}$ 


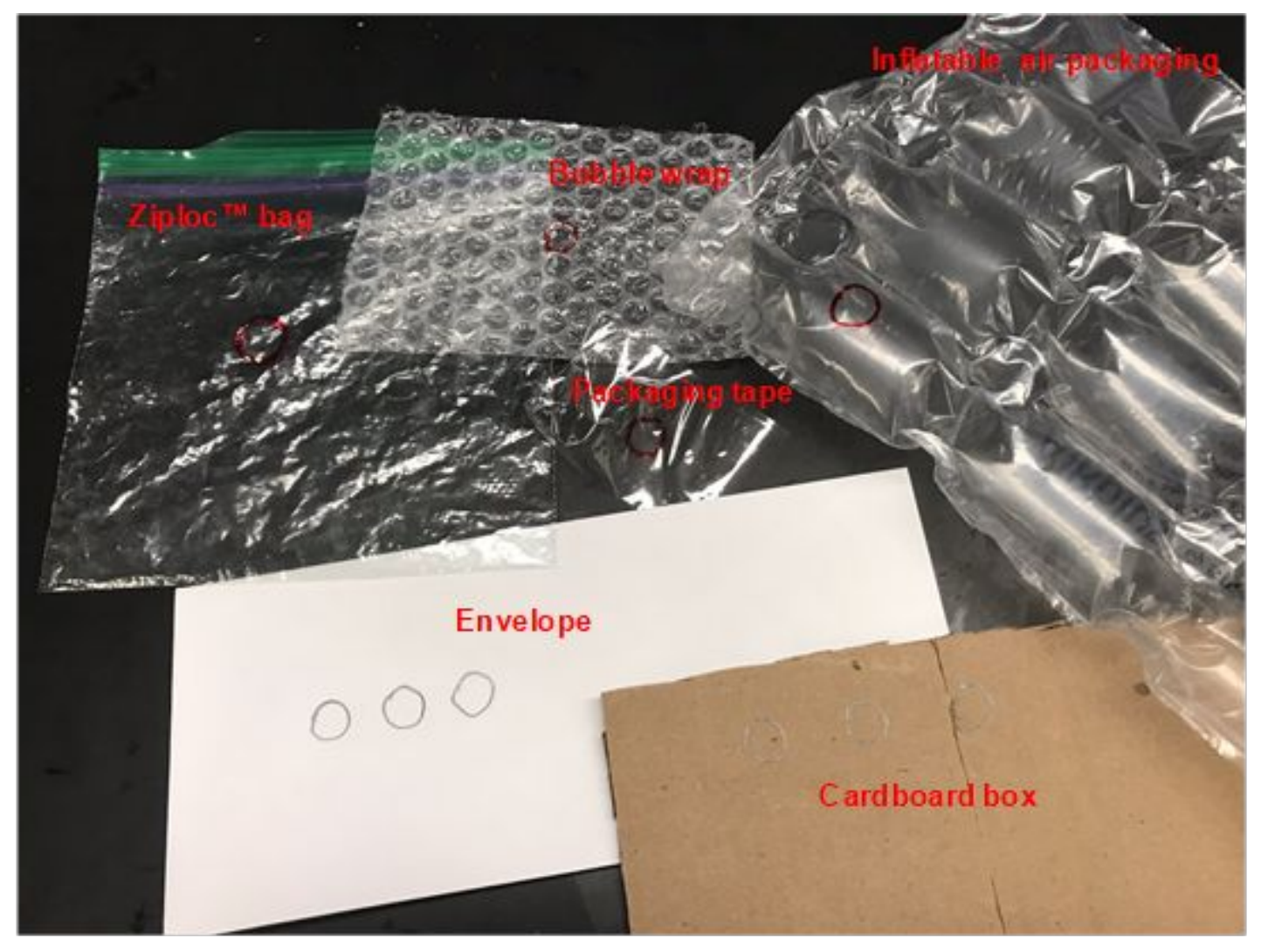

Figure S1. A photograph of different mail packages used in this study. Envelope and cardboard box represent porous materials while Ziploc bag $^{\mathrm{TM}}$, bubble wrap, and inflatable air packaging are nonporous materials. Pencil/Sharpie ${ }^{\mathrm{TM}}$ rings mark the position of deposited/dried calibrants on different packaging materials. The area within the circles swiped with OPI source. 


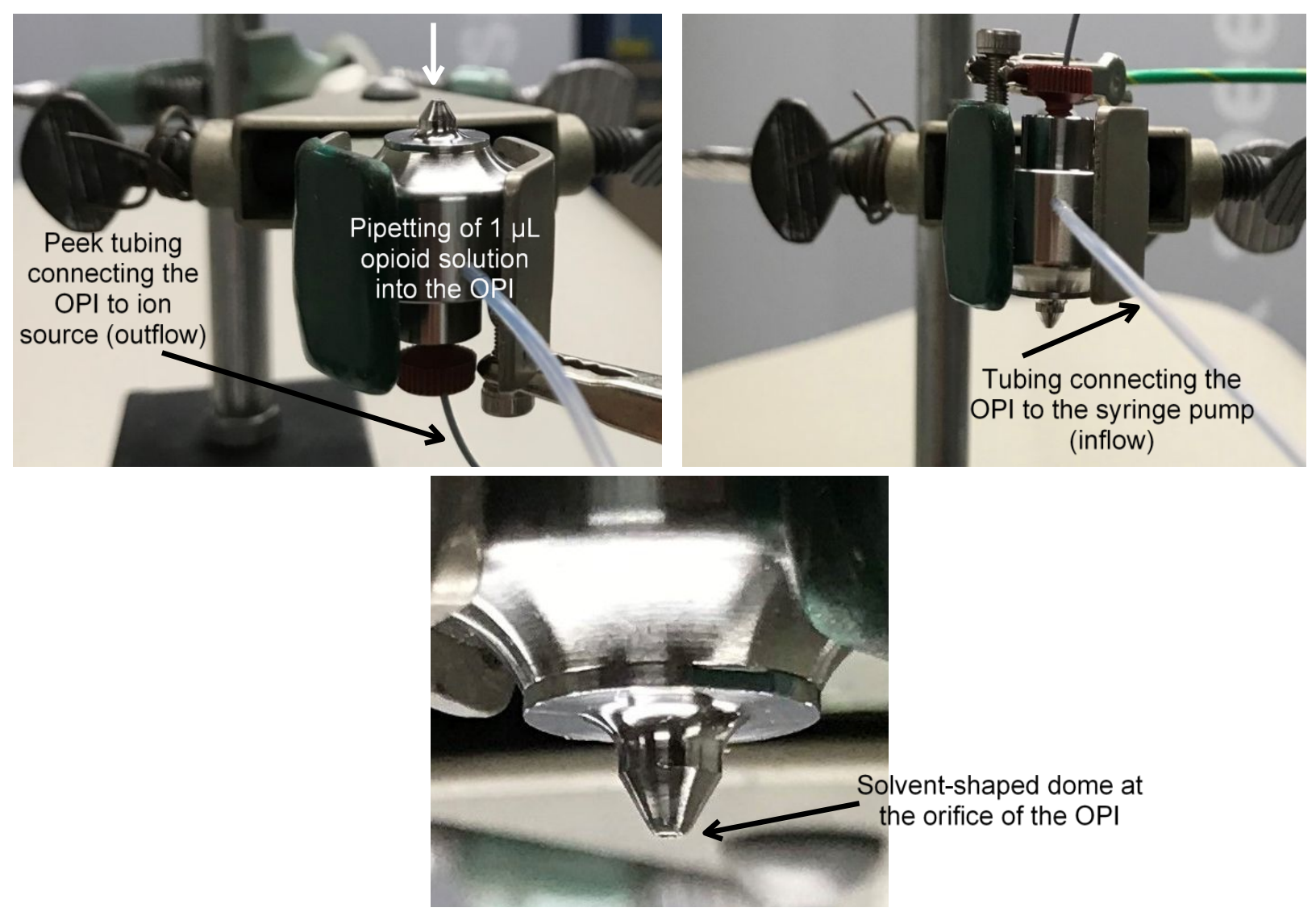

Figure S2. The OPI, upward-facing (left), where a liquid can be pipetted directly into the orifice of OPI. While the OPI, downward-facing (right) at which the solvent-shaped dome (continuously flushing) is swiped across a potentially contaminated surface. Magnified photo of the OPI showing the solvent-shaped dome (lower right). 

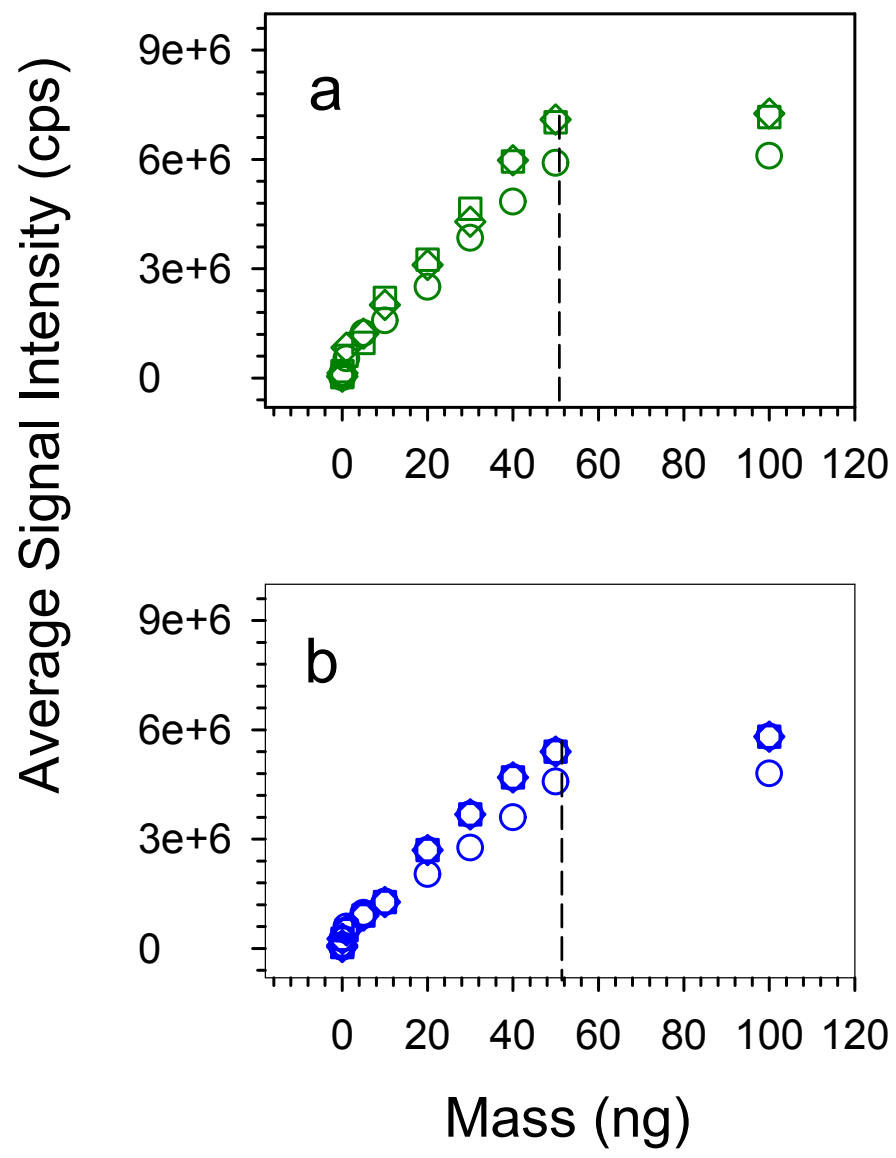

Figure S3. Average signal intensities from different deposited masses are showing that beyond $50 \mathrm{ng}$, the response is no longer linear (plateau) (a) Packaging tape and (b) Bubble wrap. The dashed line corresponds to $50 \mathrm{ng}$. 

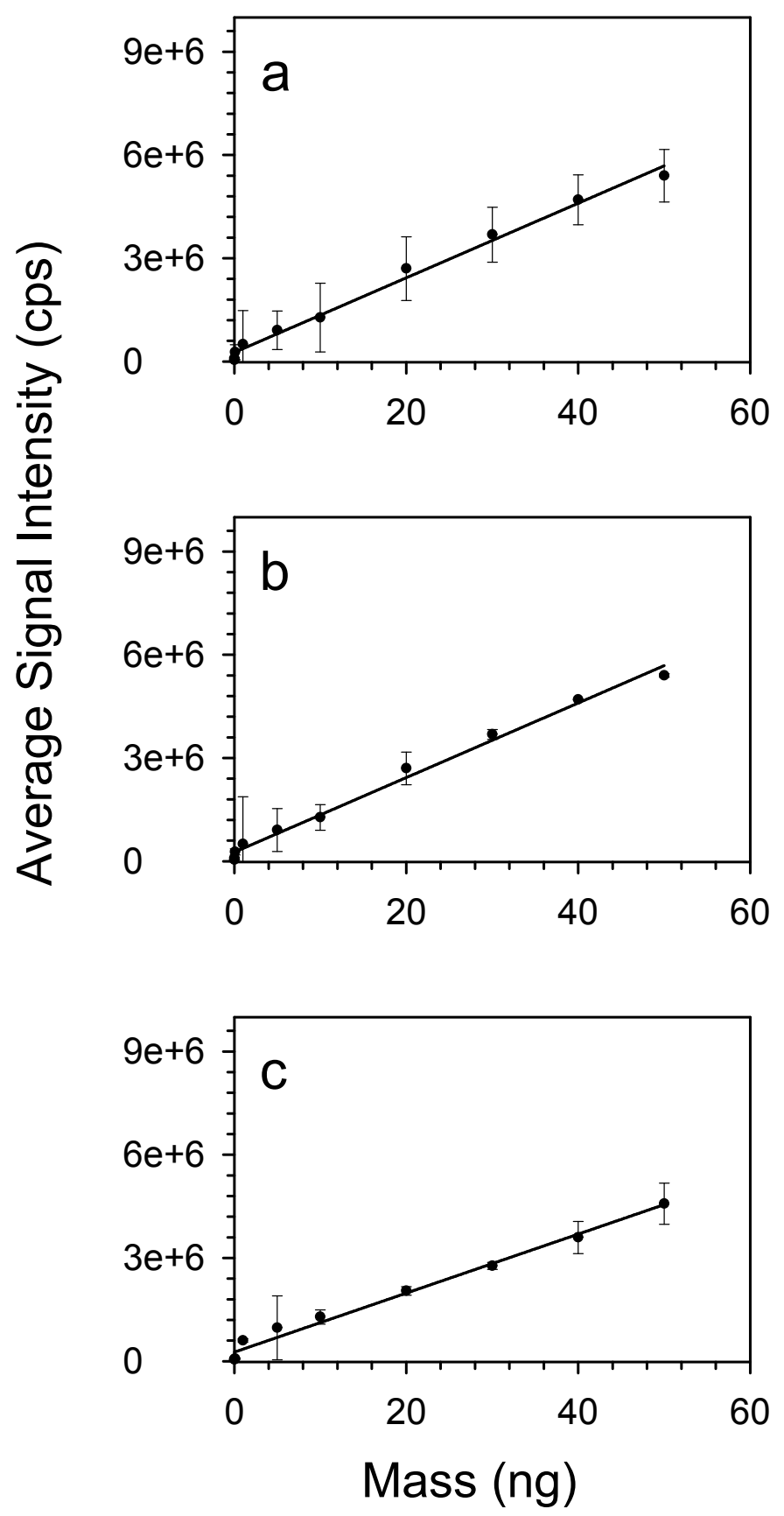

Figure S4. Quantitative analysis of different calibrants from bubble wrap. (a) Fentanyl (LOD mass $=2.5 \mathrm{ng}$ ). (b) Heroin ( $\left.\mathrm{LOD}_{\text {mass }}=2.6 \mathrm{ng}\right)$. (c) Oxycodone $\left(\mathrm{LOD}_{\text {mass }}=3.2 \mathrm{ng}\right.$ ). Error bars represent the standard deviation of three replicates. 

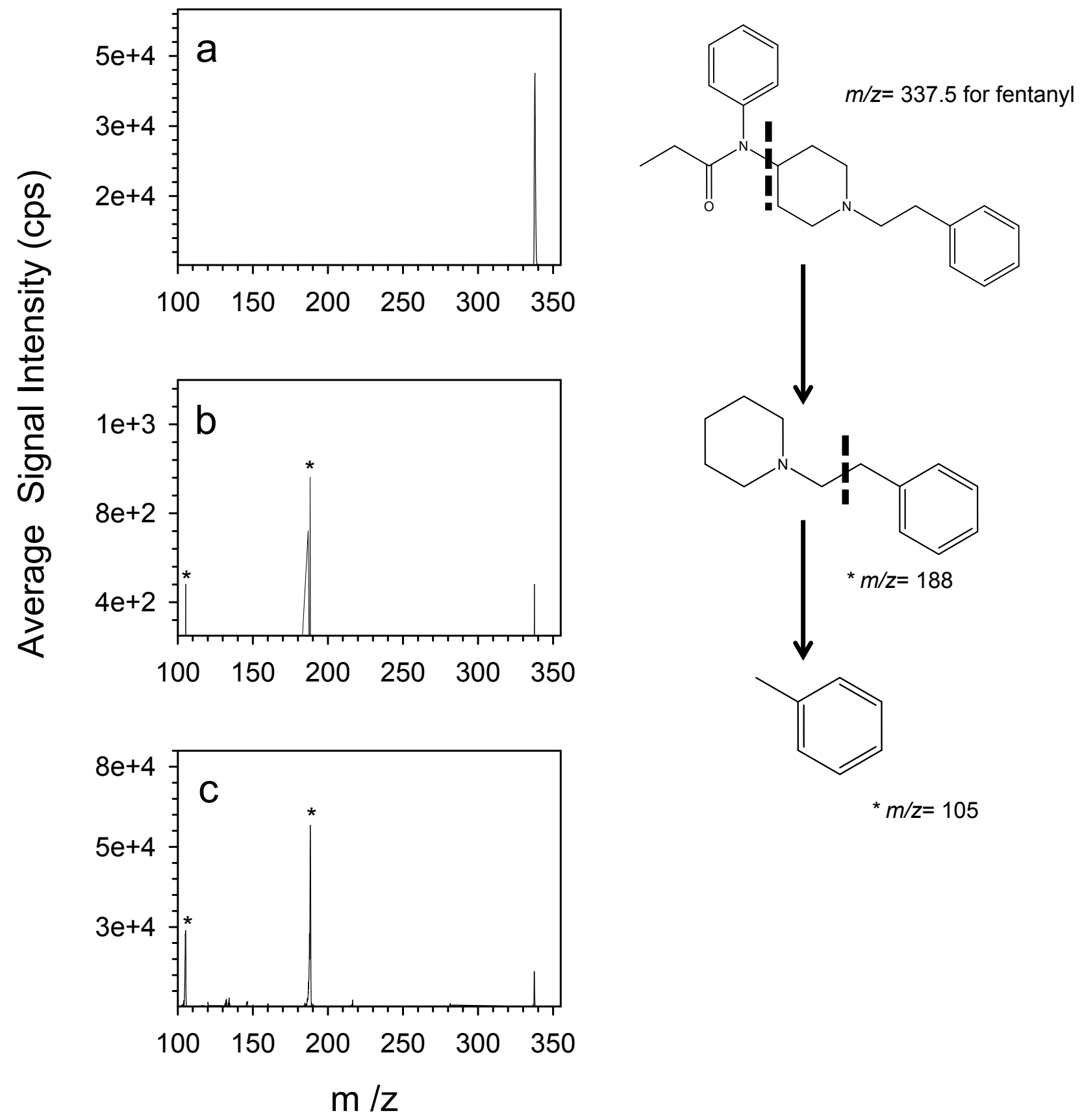

Figure S5. Mass spectra showing MS/MS fragmentation of fentanyl (precursor-ion) using collision energy ( $32 \mathrm{~V}$ ) applied to the second quadrupole. (a) The precursor-ion of fentanyl having $\mathrm{m} / \mathrm{z} 337.5$ shown at very low collision energy ( $5 \mathrm{~V})$. (b) A mass of $1 \mathrm{pg}$ from an envelope underwent tandem mass spectrometry showing the two main fragments of $m / z 188(*)$ and $\mathrm{m} / z 105\left(^{*}\right)$. (c) A mass of $1 \mathrm{ng}$ from an envelope showing the two main fragments. ${ }^{2}$ 

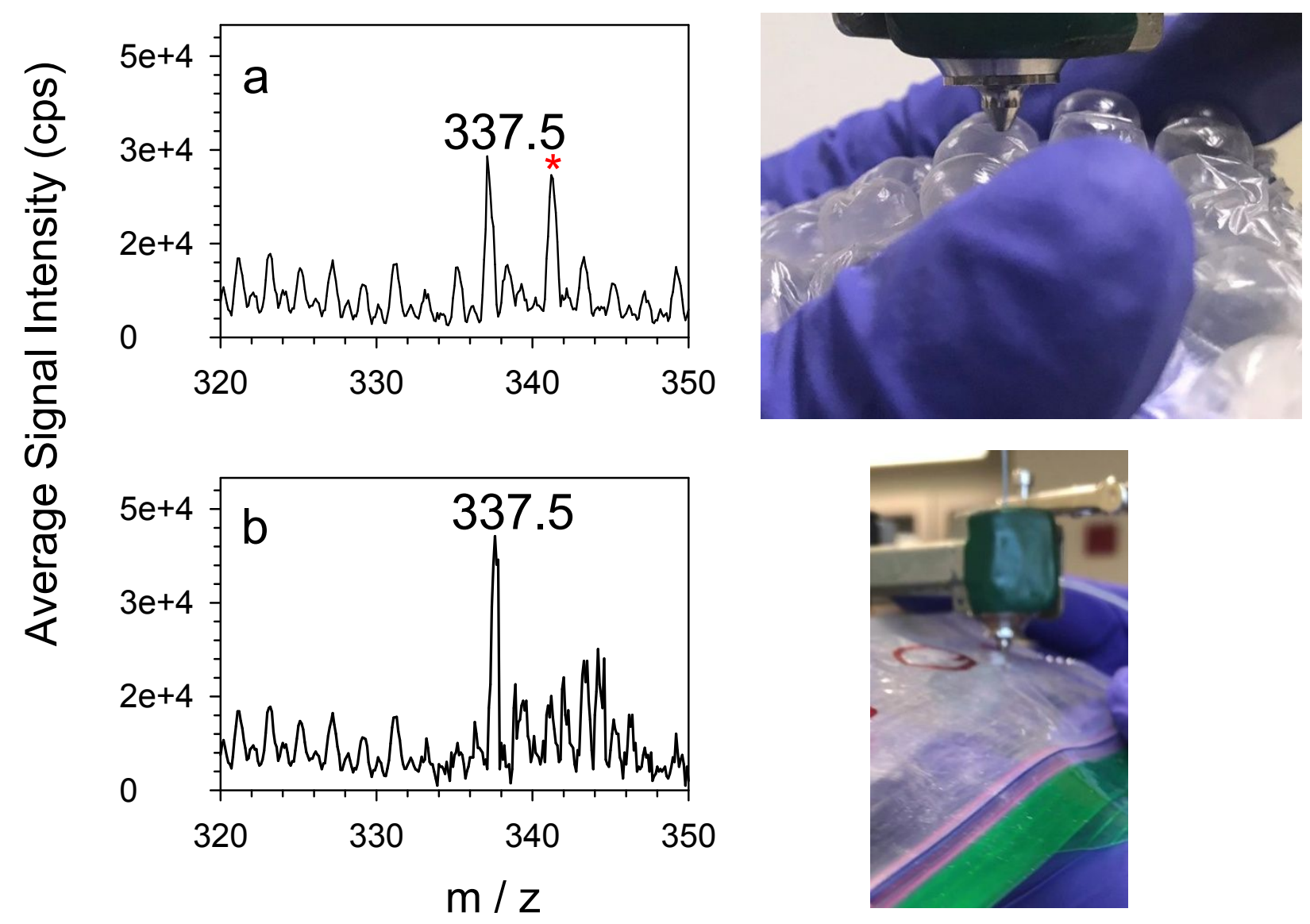

Figure S6. Non-invasive detection of opioids from (a) Bubble wrap and (b) Ziploc bag ${ }^{\mathrm{TM}}$. With bubble wrap, $1 \mathrm{ng} / \mu \mathrm{L}$ of fentanyl solution was injected inside the bubble, and the outer surface of the bubble was swiped across the OPI, showing a peak at $\mathrm{m} / \mathrm{z} 337.5$ for the singly protonated fentanyl. For the Ziploc bag ${ }^{\mathrm{TM}}$, a fentanyl solution $(1 \mathrm{ng} / \mu \mathrm{L})$ was pipetted inside the bag, and the OPI was swiped on the outer surface. The asterisk (*) represents an unknown contaminant associated with the bubble wrap. 


\section{References}

(1) Shrivastava, A.; Gupta, V. Methods for the determination of limit of detection and limit of quantitation of the analytical methods. Chron. Young Sci. 2011, 2, 21-25.

(2) Day, J.; Slawson, M.; Lugo, R. A.; Wilkins, D. Analysis of Fentanyl and Norfentanyl in Human Plasma by Liquid Chromatography-Tandem Mass Spectrometry Using Electrospray Ionization. J. Anal. Toxicol. 2003, 27, 513-516. 